\title{
Robinho Santana
}

\author{
Robinho Santana \\ Robinho Santana
}

Robinho Santana ${ }^{1}$

1 Robinho Santana, artista visual residente em Diadema, São Paulo - SP. Bacharel em Design na Universidade Morumbi e Técnico em fotografia pelo SENAC/SP. https://www.instagram.com/robinho_santana/ .E-mail: olarobinhosantana@gmail. com 


\title{
Resumo
}

Robinho Santana nasceu e cresceu em Diadema, São Paulo e hoje é artista visual, pesquisador e músico experimental, com formação acadêmica em Design e Fotografia. Seu trabalho dialoga com o dever compulsório de exprimir a sua relação com a vida e a cultura de seu povo. Em suas obras além de se reconhecer, busca a representação plural e digna da mulher e do homem negros periféricos, tornando-os protagonistas em sua arte.

\section{Palavras-chave}

Saúde mental, educação, ocupação, resistência política, afro-brasileira.

\begin{abstract}
Robinho Santana was born and raised in Diadema, São Paulo and today he is a visual artist, researcher and experimental musician, with an academic background in Design and Photography. His work dialogues with the compulsory duty to express his relationship with the life and culture of his people. In his works, in addition to recognizing himself, he seeks the plural and dignified representation of peripheral black women and men, making them protagonists in his art.
\end{abstract}

\section{Keywords}

Mental health, education, occupation, political resistance, Afro-Brazilian.

\section{Resumen}

Robinho Santana nació y se crió en Diadema, São Paulo y hoy es un artista visual, investigador y músico experimental, con formación académica en Diseño y Fotografía. Su obra dialoga con el deber obligatorio de expresar su relación con la vida y la cultura de su pueblo. En sus obras, además de reconocerse a sí mismo, busca la representación plural y digna de las mujeres y hombres negros periféricos, haciéndolos protagonistas de su arte.

\section{Palabras clave}

Palabras clave: salud mental, educación, ocupación, resistencia política, afrobrasileña. 
Em minhas obras a busca sempre foi sobre a importância de me encontrar e de fazer com que as pessoas que entrassem em contato com elas também se sentissem ali reconhecidas e representadas. Entendendo comigo mesmo e com outras pessoas ao meu redor a importância de falar sobre a nossa vida de forma plural e multidisciplinar.

A frase "somos todos iguais" nunca me caiu bem e isso eu trago para o meu trabalho de diversas formas, seja em técnicas de pintura diferentes ou outros tipos de suportes como a acrílica sobre tela, xilogravura, muralismo, serigrafia, musica ou através da diversidade de temas abordados como por exemplo a saúde mental ou a importância da educação.

Desde minha adolescência vivida em Diadema/SP onde tive minhas primeiras experiências artísticas através da musica, eu já via uma necessidade de falarmos de nós a partir de nós mesmos numa busca de mudanças, sejam elas pessoais ou sociais, muito pela influencia de ter sido criado no barulho da revolução sindicalista no grande $A B C$ onde meus pais tiveram uma grande atuação e eu vi ainda muito jovem, homens e mulheres indo as ruas lutando pelos seus direitos.

E isso, certamente, é mais uma influencia em meu trabalho de artes que eu considero sendo um jovem vindo de uma área periférica onde nossas oportunidades nada tinha haver como a carreira artística, um ato de resistência e politico. 


\section{Revista Apotheke}

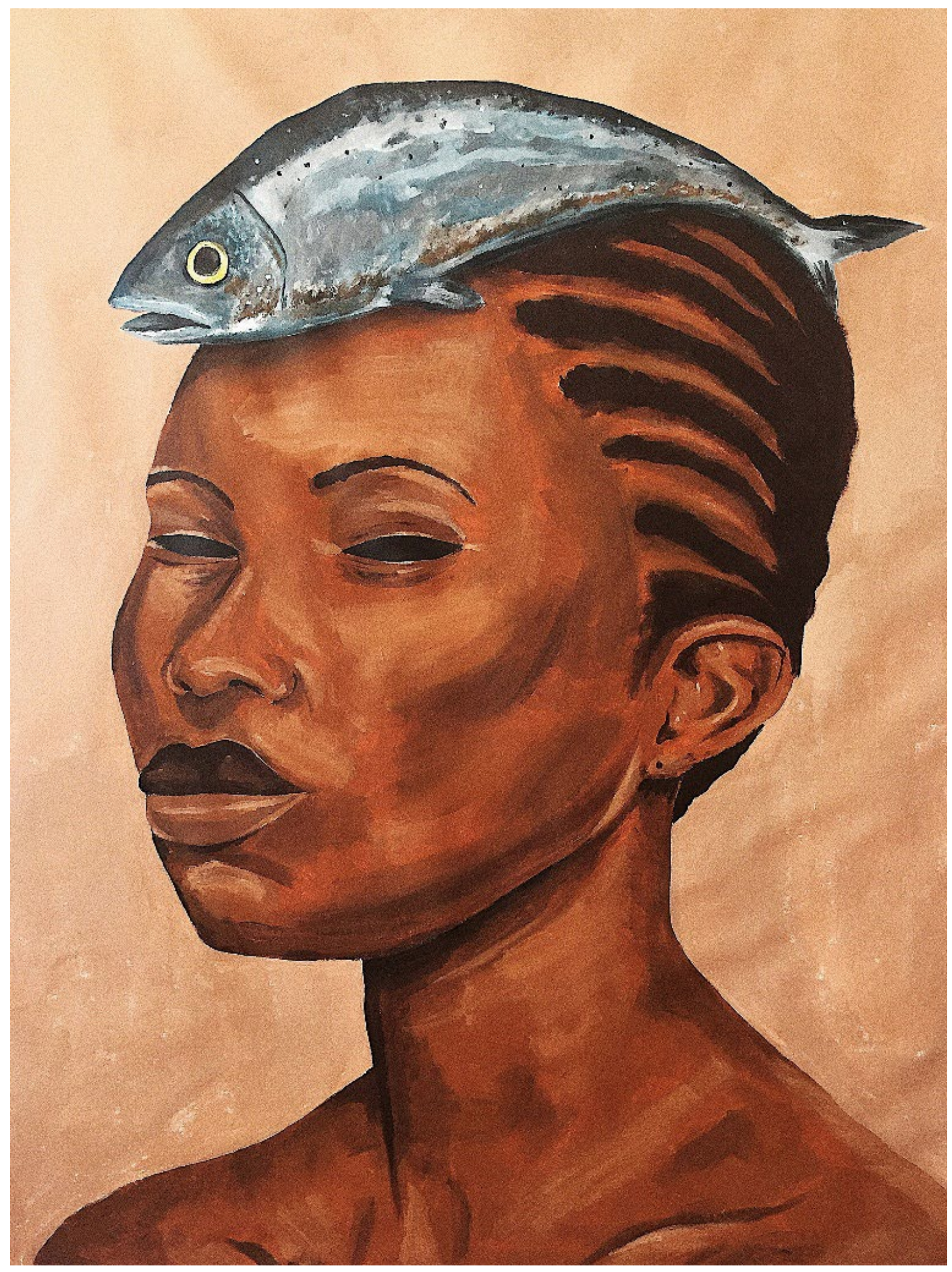

Fig. 01, Robinho Santana, O não lugar, acrílica sobre tela, 2019, 100x150cm. 


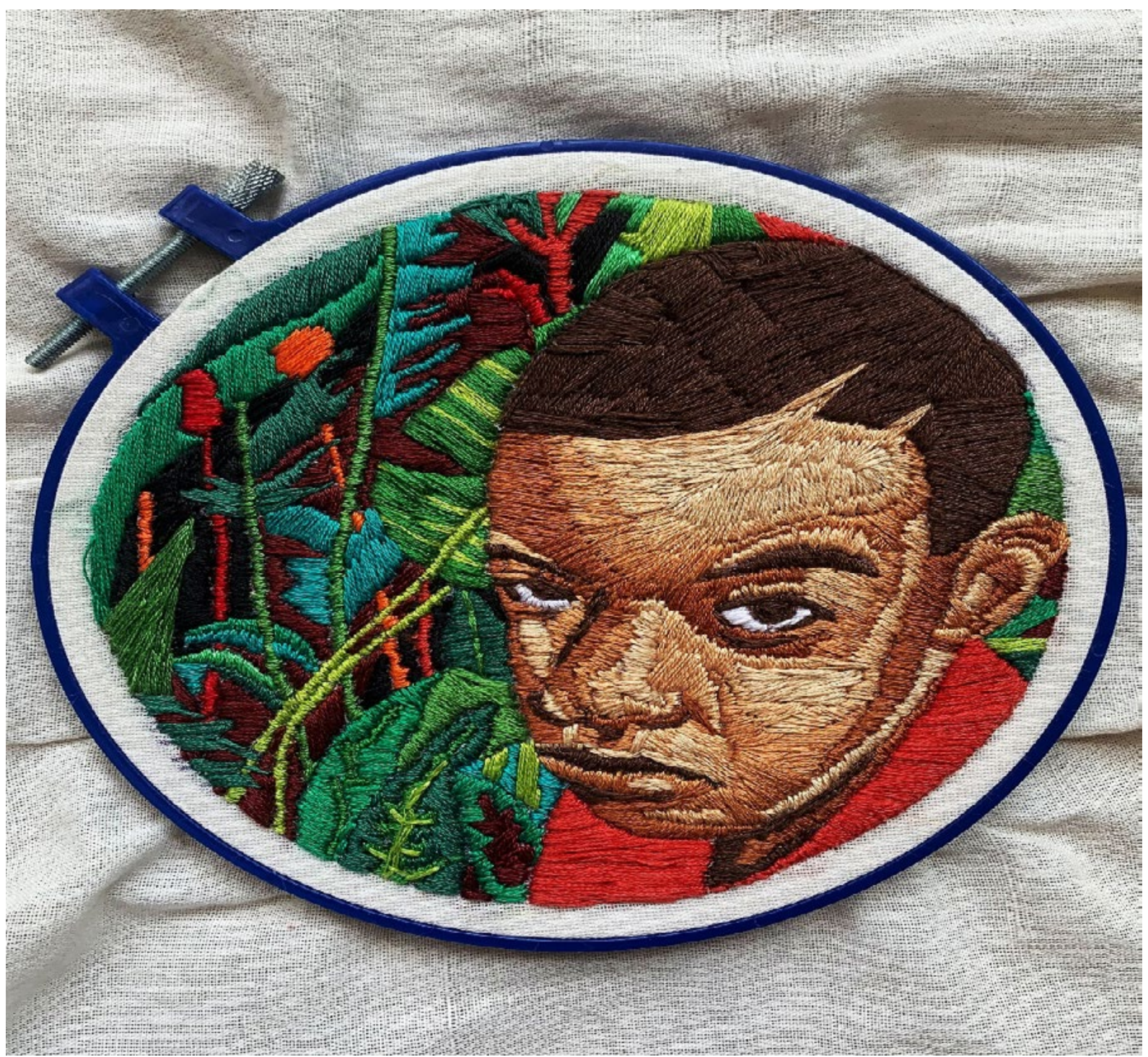

Fig. 02, Robinho Santana, Estudos sobre paciência, Bordado em algodão cru, 2021, 15×15cm. 


\section{Revista Apotheke}

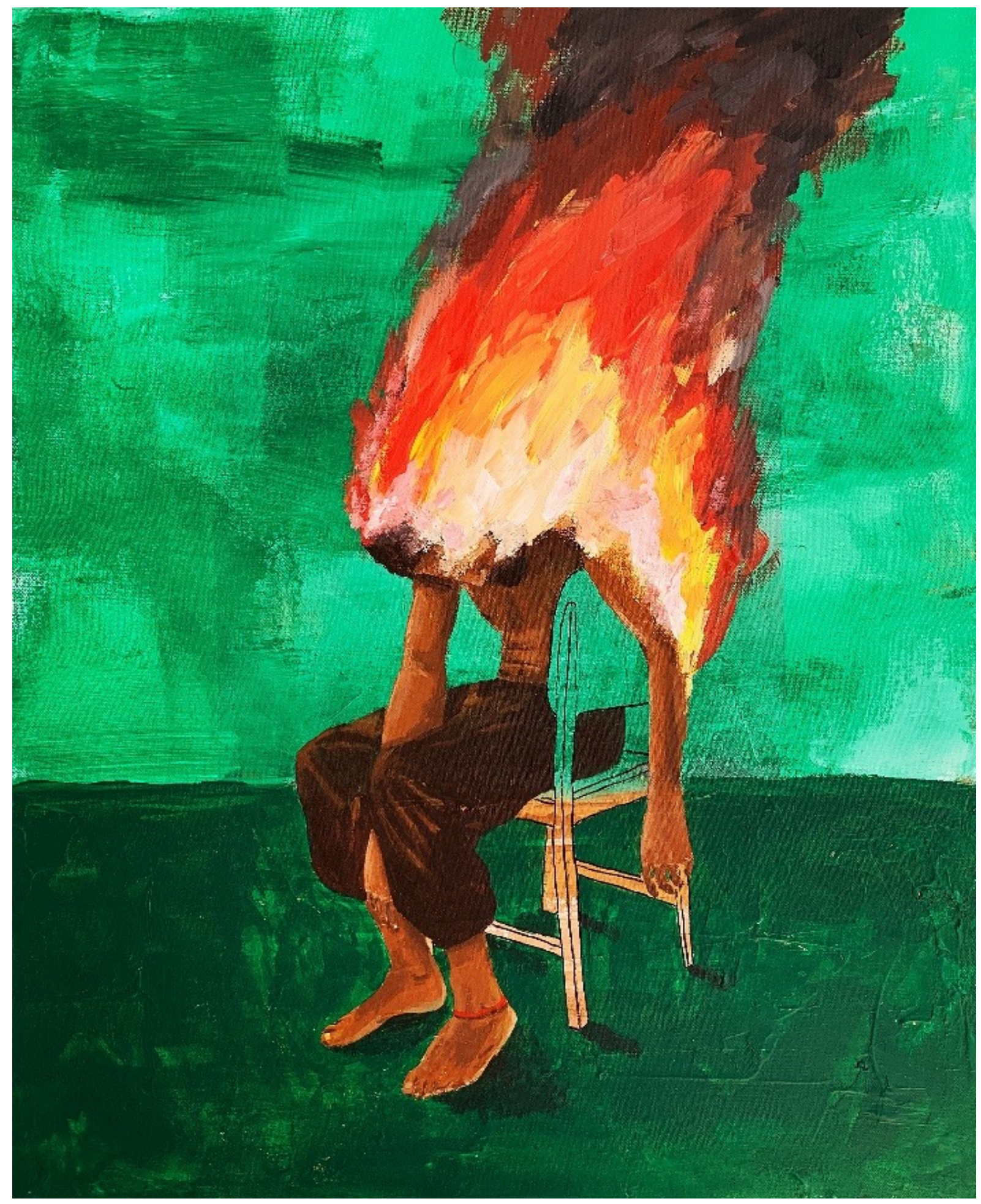

Fig. 03, Robinho Santana, Indomável, acrílica sobre tela, 2020, 40×50 cm. 


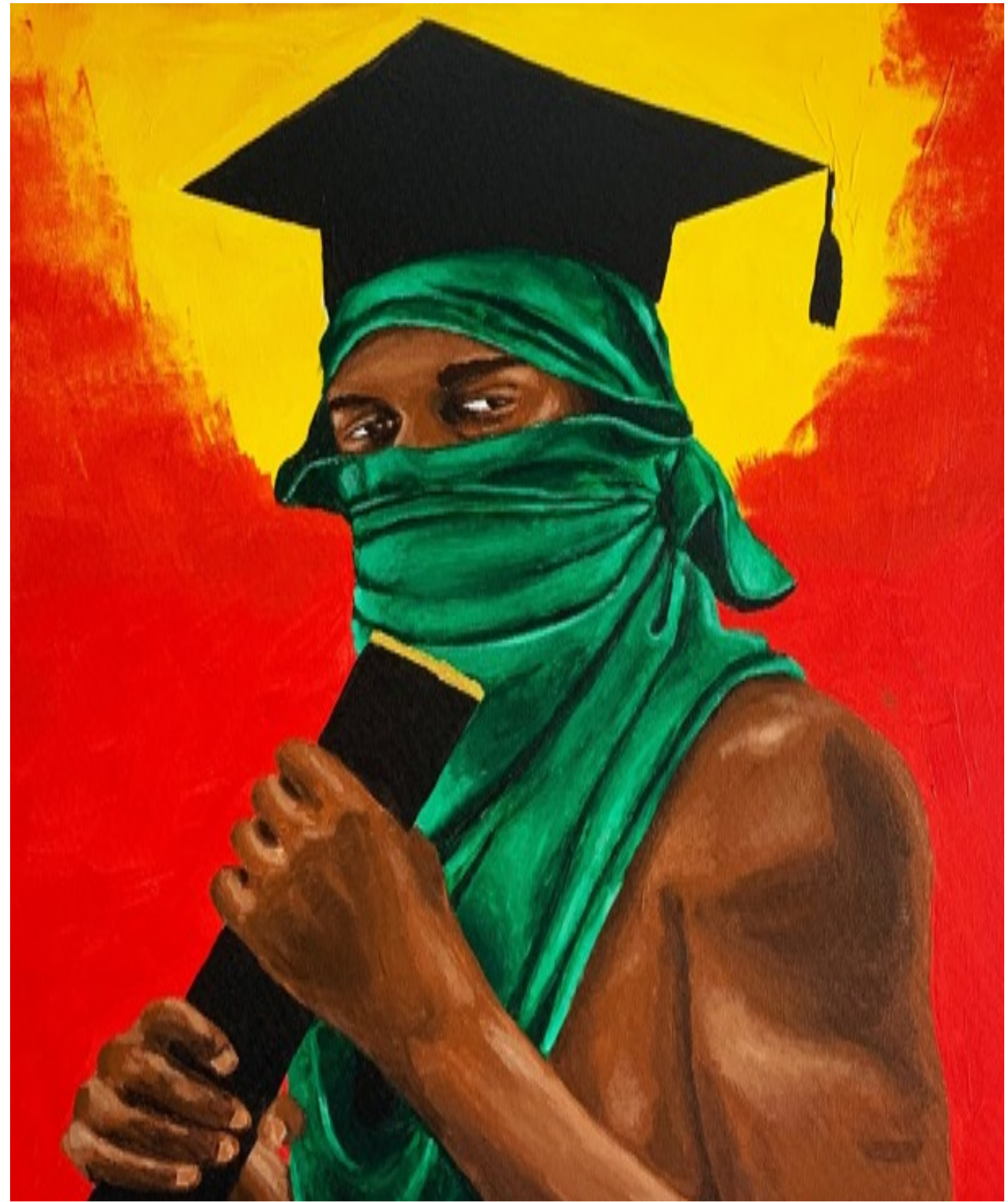

Fig. 04, Robinho Santana, Retomada, acrílica sobre tela, 2020, , 50×60 cm. 


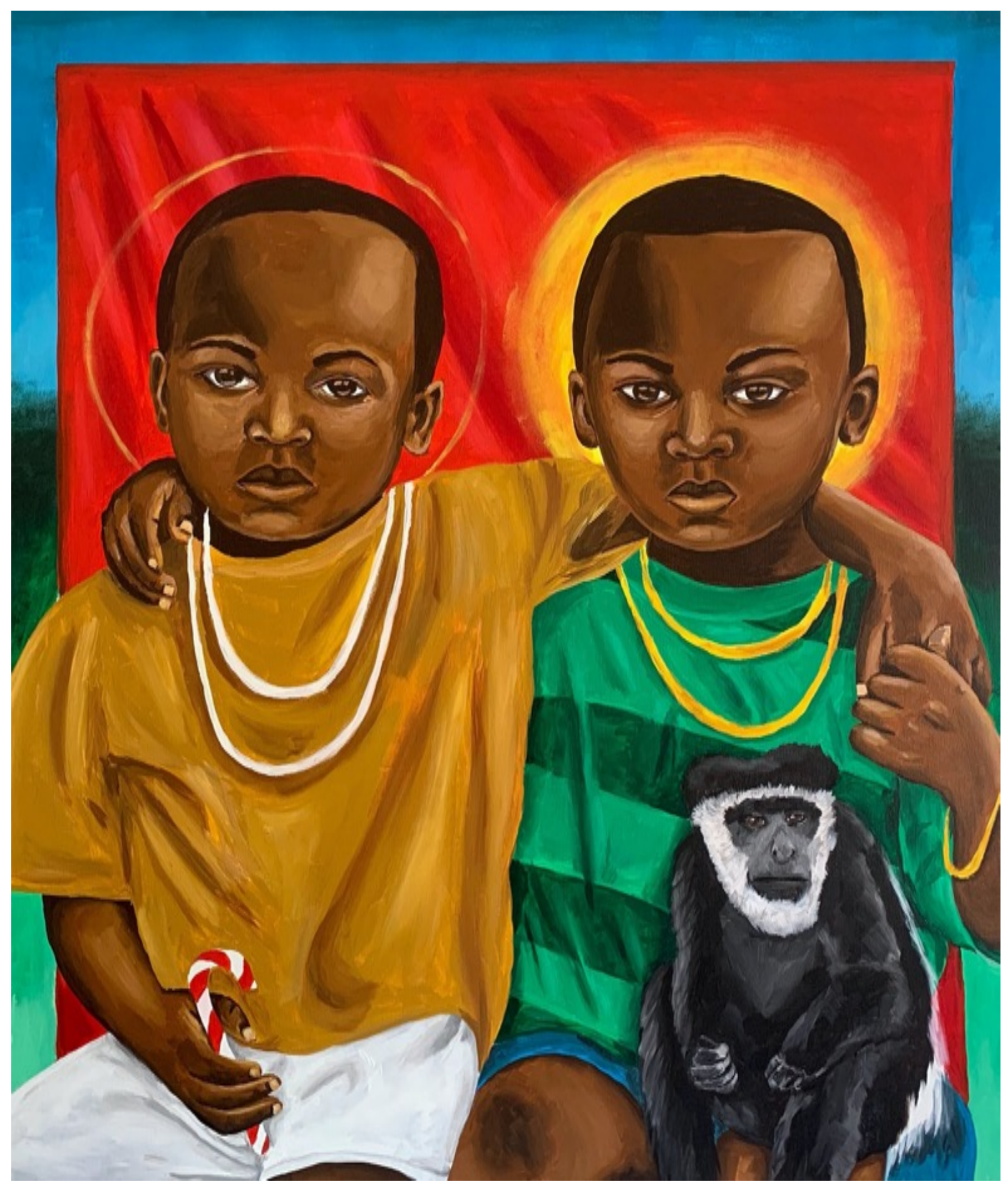

Fig. 05, Robinho Santana, Ibeji, acrílica sobre tela, 2020, 110×130 cm. 


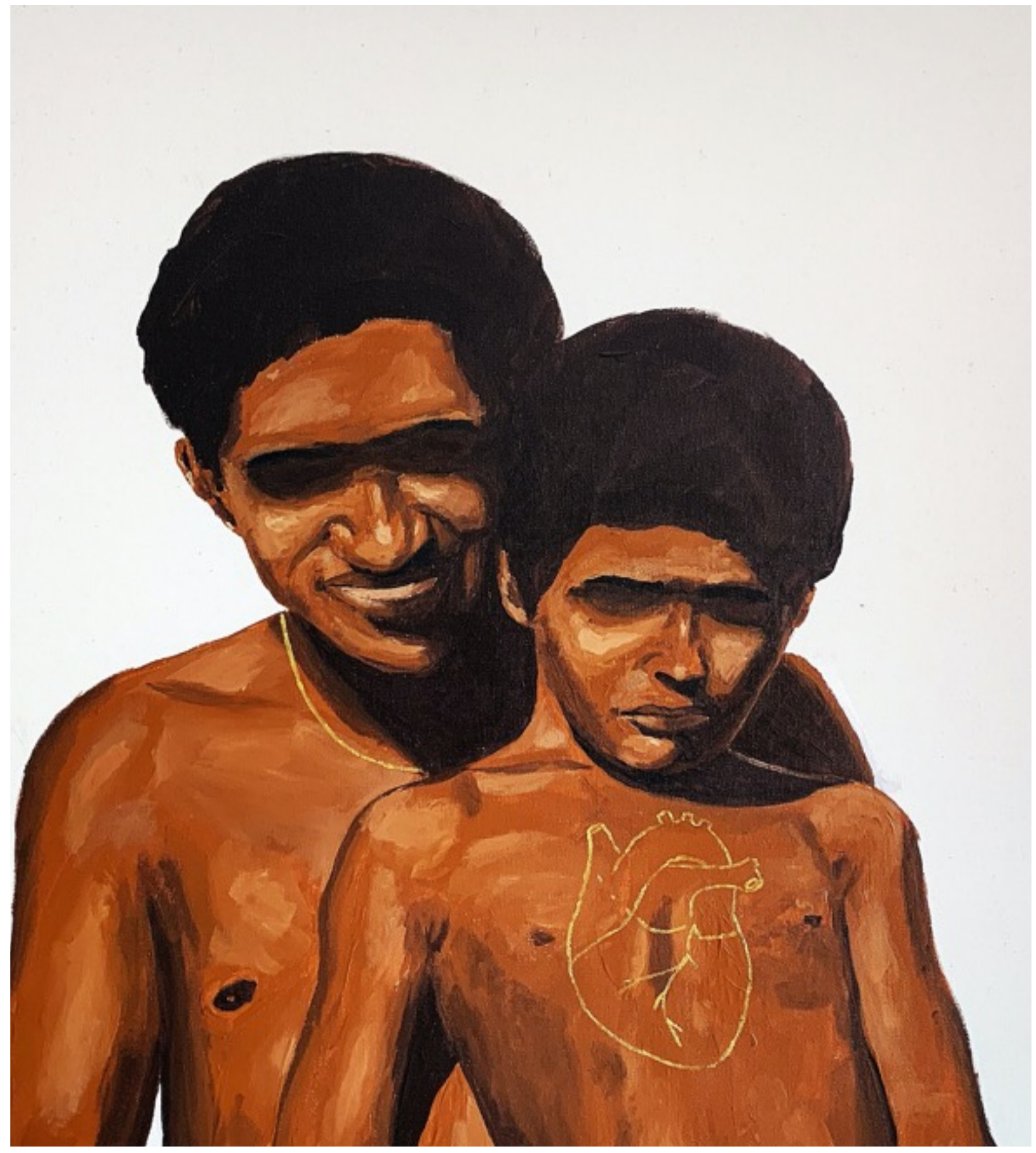

Fig. 06, Robinho Santana, Sankofa, acrílica sobre tela, 2020, 50×60 cm. 


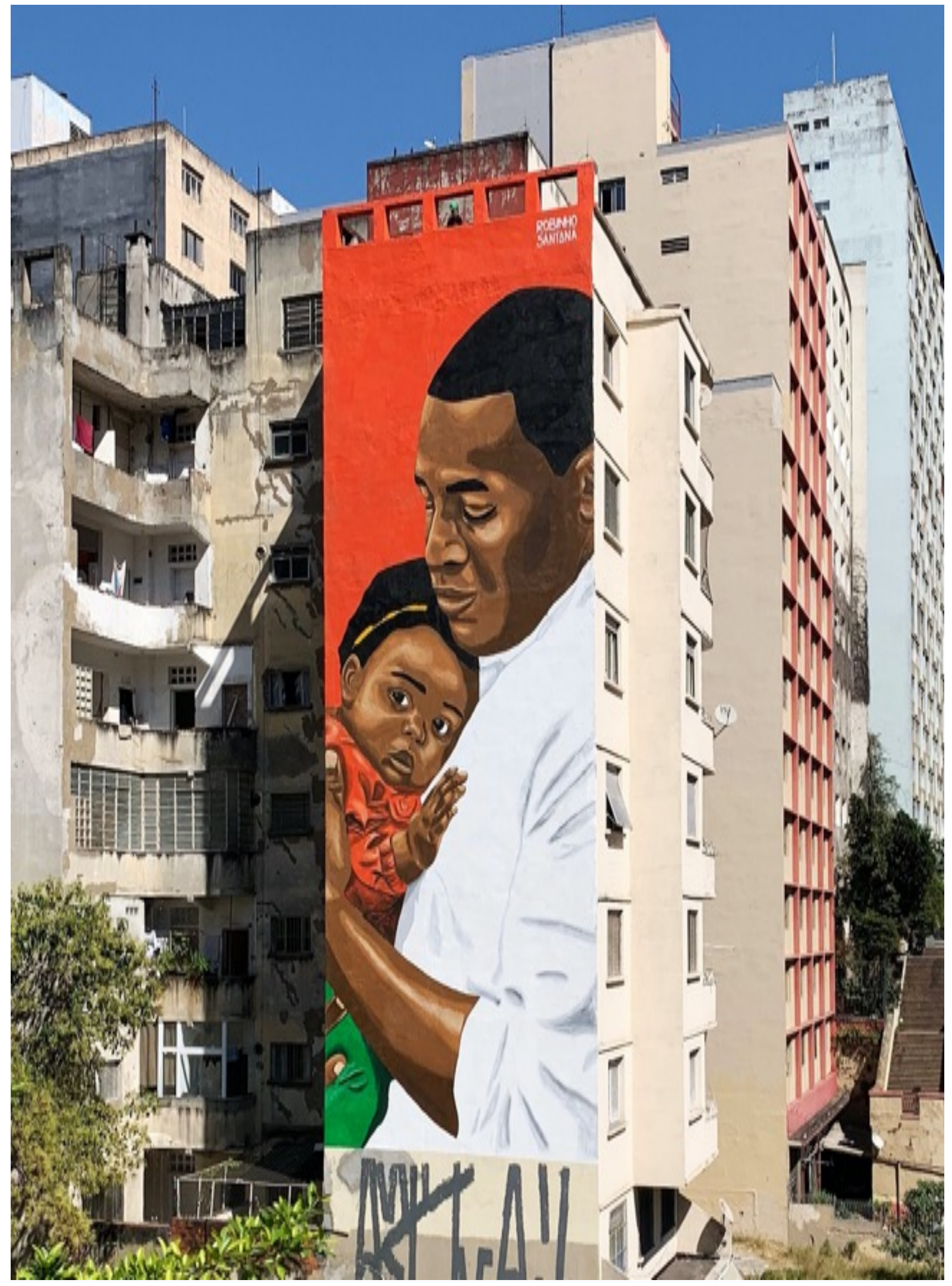

Fig. 07, Robinho Santana, ...importância da paternidade..., mural localizado em são Paulo - Bela Vista, 2020. 


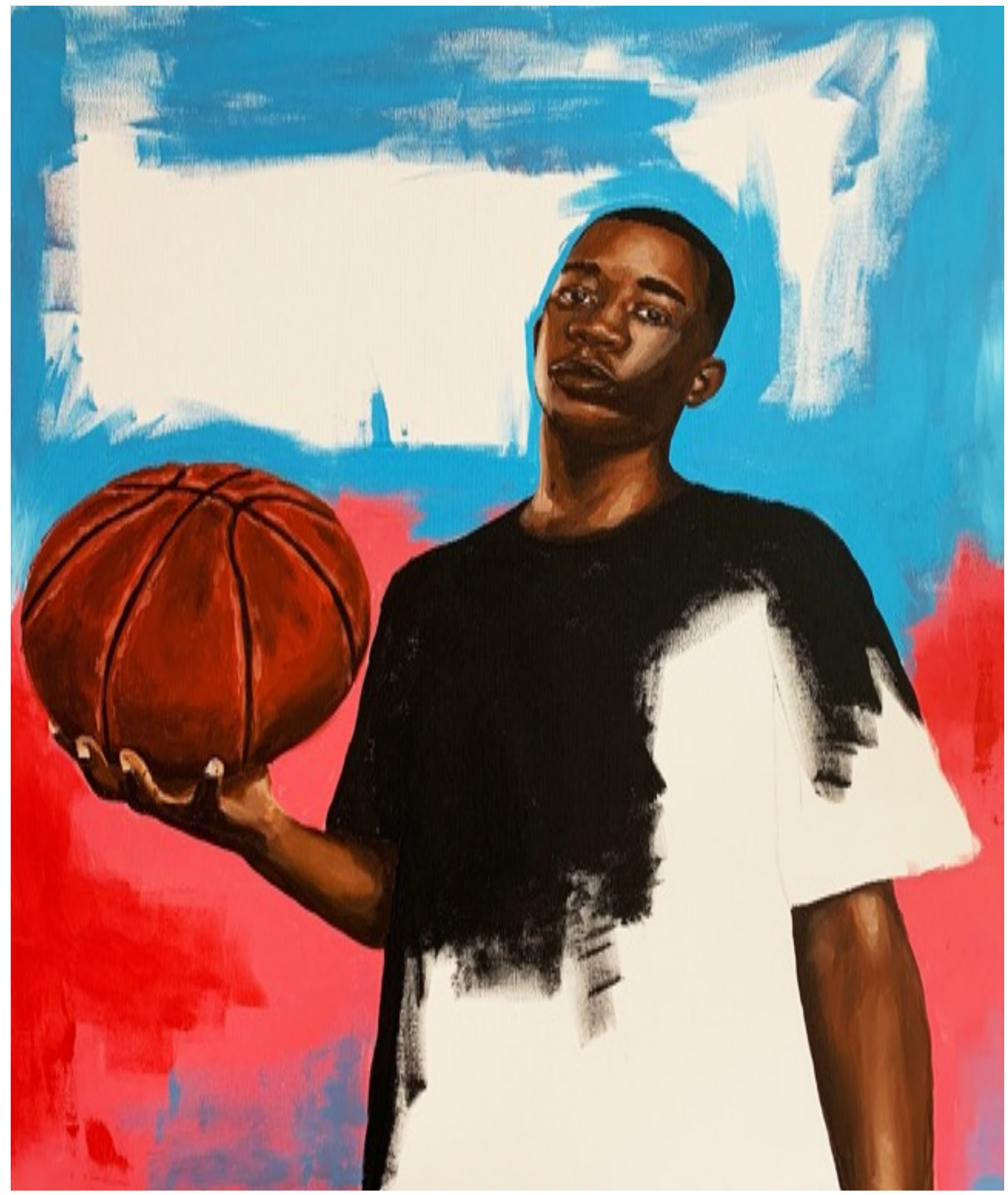

Fig. 08, Robinho Santana, Untitled, Acrílica sobre tela, 2021, 80×100 cm. 


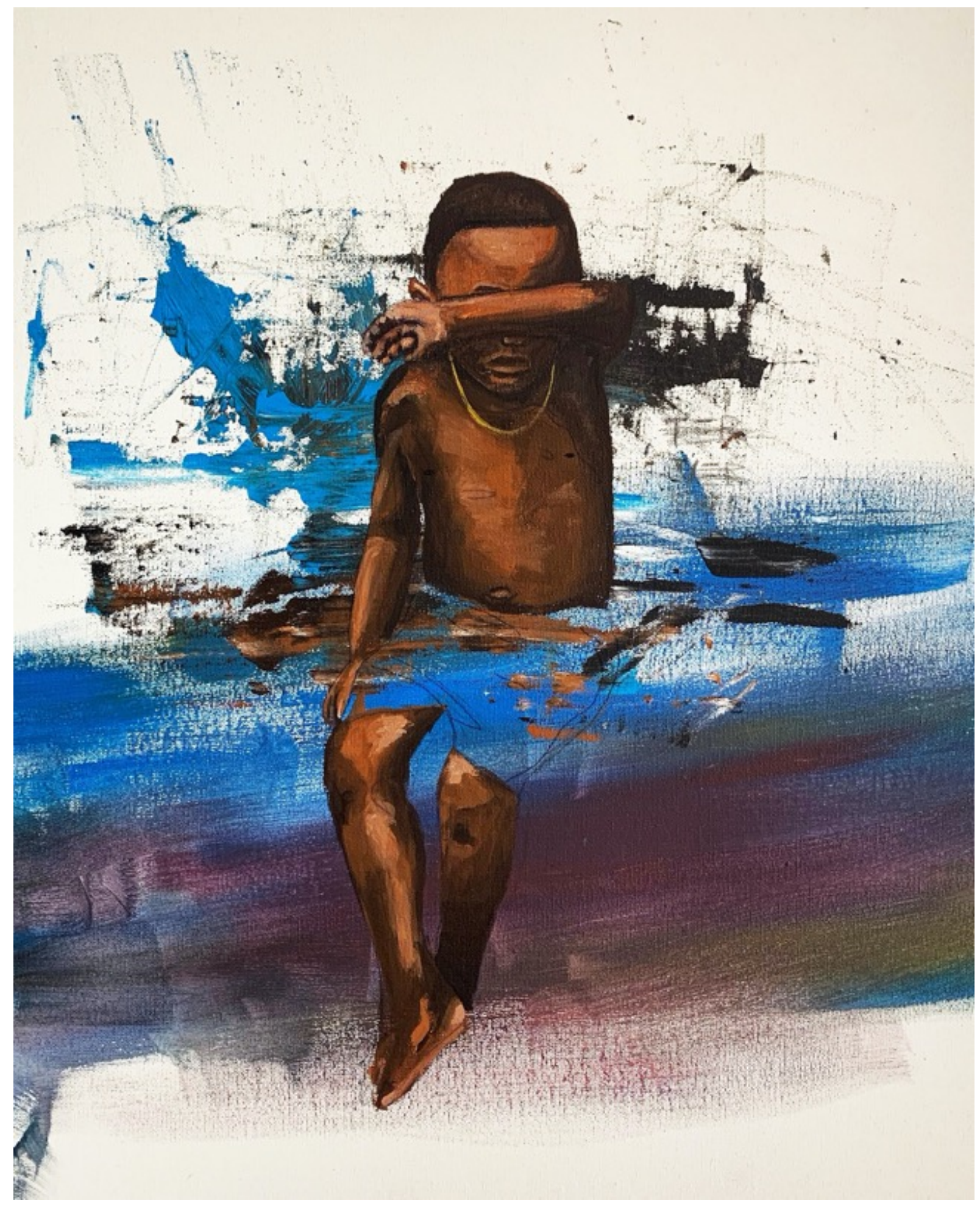

Fig. 09, Robinho Santana, O que eu queria era um dia não ter que falar sobre isso mais uma vez, Acrílica sobre tela, 2021, 40×50 cm. 